risk factor for recurrence of febrile seizures. Children with recurrences have higher temperatures than those without, supporting the theory of a febrile seizure threshold dependent on the height of the temperature.

Prediction of febrile seizures in siblings was studied prospectively in 129 children with FC at Sophia Children's Hospital, Rotterdam, The Netherlands (van Esch A, Steyerberg EW, van Duijn CM et al. Eur I Pediatr April 1998;157:340$344)$. The risk of FS in siblings was $10 \%$, more than twice the population risk (4\%). The overall risk of FS in first degree relatives of FS probands was $7 \%$. The risk was increased to $16 \%$ in siblings with recurrent FS and $25 \%$ in siblings with an affected parent. A polygenic mode of inheritance seemed likely. A prediction model based on three risk factors was developed. The risk factors are: 1) FS in the parents; 2) age at first FS of proband under 1 year; and 3) FS recurrence in the proband. The risk of FS in siblings of FS probands may increase to $46 \%$ with 2 or 3 risk factors present and it falls to less than $10 \%$ if a sibling is unaffected up to 3 years of age.

\title{
CHILDHOOD EPILEPSY TREATMENT STRATEGIES
}

Treatment strategies employed in 494 children with various seizure types and remission frequencies were studied prospectively at multiple hospital centers in the Netherlands. In $142(29 \%)$ treatment was initially withheld, and after 2 years $17 \%$ were still untreated, none suffering serious complications. Of 416 treated with AEDs, $88 \%$ received valproic acid or carbamazepine initially, and $40 \%$ did not respond successfully. Reasons for treatment failures included recurrent seizures $(28 \%)$, and intolerable side effects $(11 \%)$. Rashes occurred in $15(4 \%), 14$ with carbamazepine ( $10 \%$ of all children who received carbamazepine). The chance of achieving remission was negatively associated with the number of AED regimens tried. Alternative AEDs included phenytoin, phenobarbital, ethosuximide, and vigabatrin. If 3 regimens had failed, the chance of remission with alternative therapy was only $10 \%$. The epilepsy was considered intractable in only $7 \%$. A distinction was made between acceptable control, with low seizure frequency or severity, and intractable epilepsy. (Carpay HA, Arts WFM, Geerts AT et al. Epilepsy in childhood. An audit of clinical practice. Arch Neurol May 1998;55:668-673). (Reprints: Willem FM Arts MD, PhD, Department of Child Neurology, University Hospital-Sophia Children's Hospital, Dr Molewaterplein 60, 3015 GJ Rotterdam, The Netherlands).

COMMENT. The initial choice of AED therapy, mainly valproic acid or carbamazepine, fails to control childhood epilepsies in $40 \%$ of cases. Alternative therapies are necessary because of seizure recurrences or side effects, especially skin rash, a frequent complication of carbamazepine treatment. Newer AEDs such as gabapentin (Neurontin ${ }^{\circledR}$ ), with a relatively low incidence of side effects, should increase the remission frequency of childhood epilepsies, especially partial seizures. In one large study of gabapentin as add-on therapy of 705 adult patients, skin rash was reported in only $0.5 \%$ compared to a $10 \%$ incidence with carbamazepine (Progress in Pediatric Neurology III, 1997; pp122-125).

\section{BILATERAL RASMUSSEN CHRONIC ENCEPHALITIS}

Two Peruvian brothers, ages 16 months and 5 years, with alternating epilepsia partialis continua are reported from the Montreal Neurological Hospital, McGill University, Canada, and Loyola University, Chicago. Seizures began at 6 months in one child and at 4 months in the other, both associated with a febrile illness. Severe psychomotor regression and cerebral atrophy developed rapidly. A brain biopsy in one child revealed chronic encephalitis with changes compatible 